\title{
Time and Frequency Domain Analysis of the Linear Fractional-order Systems
}

\author{
Manisha K.Bhole \\ Instrumentation Dept. \\ Bharati Vidyapeeth College of Engg. \\ Navi Mumbai, India
}

\author{
Mukesh D. Patil \\ Research Scholar, \\ IDP in Systems \& Control Engg. \\ Indian Institute of Technology Bombay, Mumbai, India
}

\author{
Vishwesh A. Vyawahare \\ Research Scholar, \\ IDP in Systems \& Control Engg. \\ Indian Institute of Technology Bombay, Mumbai, India
}

\begin{abstract}
Recent years have seen a tremendous upsurge in the area related to the use of Fractional-order (FO) differential equations in modeling and control. FO differential equations are found to provide a more realistic, faithful, and compact representations of many real world, natural and manmade systems. FO controllers, on the other hand, have been able to achieve a better closed-loop performance and robustness, than their integer-order counterparts. In this paper, we provide a systematic and rigorous time and frequency domain analysis of linear FO systems. Various concepts like stability, step response, frequency response are discussed in detail for a variety of linear FO systems. We also give the state space representations for these systems and comment on the controllability and observability. The exercise presented here conveys the fact that the time and frequency domain analysis of FO linear systems are very similar to that of the integer-order linear systems.
\end{abstract}

Keywords- Fractional-order systems, fractional calculus, stability analysis.

\section{INTRODUCTION}

The mathematical modeling of FO systems and processes, based on the description of their properties in terms of Frac- tional Derivatives (FDs), leads to differential equations of in- volving FDs that must be analyzed. These are generally termed as Fractional Differential Equations (FDEs). The advantages of fractional calculus have been described and pointed out in the last few decades by many authors in [1], [2], [3], [8], [9], [24]. The latest and very exhaustive literature survey about the FC and FO systems is given in [17]. It has been shown that the FO models of real systems (especially distributed parameter type and memory type) are more adequate than the usually used Integerorder (IO) models.

Fractional Derivatives (FDs) provide an excellent instrument for the description of memory and hereditary properties of various materials and processes. This is the main advantage over the IO models, which possess limited memory. The advantages of FDs become apparent in applications including modeling of damping behaviour of visco-elastic materials, cell diffusion processes [8], transmission of signals through strong magnetic fields, modeling mechanical and electrical properties of real materials, as well as in the description of rheological properties of rocks, and in many other fields [25].

In feedback control, by introducing proportional, integral and derivative control actions of the form $\mathrm{s}^{\alpha}, 1 / \mathrm{s}^{\alpha}, \alpha \in \mathrm{R}^{+}$, we can achieve more robust and flexible design methods to satisfy the controlled system specifications. Studies have shown that an FO controller can provide better performance than integer order (IO) controller.

The paper is organised as follows : Section II and III give special functions and definitions of fractional calculus theory respectively. Section IV defines linear FO system in general. Section $\mathrm{V}$ describes the stability analysis of fractional-order systems, Section VI explains the representations of fractional-order systems and in Section VII analytical results of FO systems are given with the conclusion in Section VIII.

\section{Special Functions of Fr actional Calculus} (FC)

Some special functions need to be used in Fractional Calculus (FC). These functions play important role in the theory of FC and in the theory of fractional differential equations (FDEs).

\section{A. Gamma Function}

One of the most basic functions of FC is Euler's gamma function $\Gamma(\mathrm{z})$, which generalizes the factorial function $\mathrm{z}$ ! and allows $\mathrm{z}$ to take also non-integer and even complex values [10]. The gamma function $(\Gamma(\mathrm{z}))$ is given by the following expression,

$$
\Gamma(z)=\int_{0}^{\infty} e^{-u} u^{z-1} d u
$$


Note that when $\mathrm{z} \in \mathrm{Z}^{+}$we have $\Gamma(\mathrm{z}+1)=\mathrm{z}$ !

\section{B. Mittag-Leffler Function}

The exponential function $\mathrm{e}^{\mathrm{Z}}$ plays a very important role in the theory of integer order differential equations. Its 1 parameter generalization function for a complex number $\mathrm{z}$ is given by [10],

$$
E_{\alpha}(z)=\sum_{k=0}^{\infty} \frac{z^{k}}{\Gamma(\alpha k+1)},
$$

The 2 parameter function of the ML function, which is also important in FC is defined as,

$$
E_{\alpha, \beta}(z)=\sum_{k=0}^{\infty} \frac{z^{k}}{\Gamma(\alpha k+\beta)},(\alpha>0, \beta>0) .
$$

This basic definition is very useful in deriving the response of an FO system to any forcing function, for example, step response, ramp response.

\section{DEFINITIONS FOR FRACTIONAL-DIFFERINTEGRALS}

The three equivalent definitions [6],[10] most frequently used for the general fractional derivatives (FD) are the Grunwald-Letnikov (GL) definition, the Riemann-Liouville and the Caputo definition [10]. In all the definitions below, the function $f(t)$ is assumed to be sufficiently smooth and locally integrable.

1) The Grunwald-Letnikov definition of fractional-order $\alpha \in \mathfrak{R}^{+}$using Podlubny's limited memory principle [4] is given by

$$
{ }_{a} D_{t}^{\alpha} f(t)=\lim _{h \rightarrow 0} h^{-\alpha} \sum_{j-0}^{[(t-a) / h]}(-1)^{j \alpha} C_{j} f(t-j h),
$$

where [.] means the integer part and ${ }^{\alpha} C_{j}$ is the binomial coefficient.

2) The Riemann-Liouville definition obtained using the Riemann-Liouville integral is given as,

$$
{ }_{a} D_{t}^{\alpha} f(t)=\frac{1}{\Gamma(n-\alpha)} \frac{d^{n}}{d t^{n}} \int_{a}^{t} \frac{f^{n}(\tau)}{(t-\tau)^{\alpha-n+1}} d \tau,
$$

for $(\mathrm{n}-1<\alpha<\mathrm{n})$ and $\Gamma($.$) is the Gamma function.$

3) The Caputo definition can be written as,

$$
{ }_{a} D_{t}^{\alpha} f(t)=\frac{1}{\Gamma(\alpha-n)} \int_{a}^{t} \frac{f^{n}(\tau)}{(t-\tau)^{\alpha-n+1}} d \tau,
$$

for $(\mathrm{n}-1<\mathrm{r}<\mathrm{n})$, where $f^{n}(t)$ is the $\mathrm{n}^{\text {th }}$ order derivative of the function $f(t)$. Since we deal with causal systems in the control theory, the lower limit is fixed at $a=0$ and for the brevity it will not be shown in this paper. We see that the Caputo definition is more restrictive than the RL. Nevertheless, it is preferred by engineers and physicists because the FDEs with Caputo derivatives have the same initial conditions as that for the integer-order differential equations. Note that the FDs calculated using these three definitions coincide for an initially relaxed function $((f(t=0)=0)$.

\section{LINEAR FRACTIONAL-ORDER SYSTEMS}

A general linear FO system is given by the FO transfer function as :

$$
G(s)=\frac{Y(s)}{U(s)}=\frac{\sum_{k=0}^{m} b_{k}\left(s^{\alpha}\right)^{k}}{\sum_{k=0}^{n} a_{k}\left(s^{\alpha}\right)^{k}},
$$

where $a_{n}=1, m>n, Y(s)$ and $U(s)$ are the Laplace transforms of the output $y(t)$ and input $u(t)$ respectively. It can be represented by the block diagram as shown in Fig.(1).

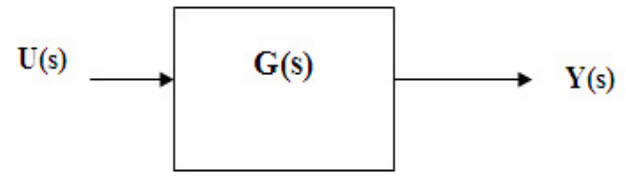

Figure 1. Block diagram representation of linear FO system.

Fig.(2) represents the general block diagram of a closedloop FO system with $Y(s)$ and $U(s)$ are the Laplace transforms of the output $y(t)$ and input $u(t)$ respectively, $k$ is the gain, $G(s)$ is the system transfer function, and $H(s)$ is the feedback component. $Y(s)$ and $U(s)$ are not usual polynomials but are pseudo-polynomials with fractional-orders. In this work we have taken unity feedback for all examples.

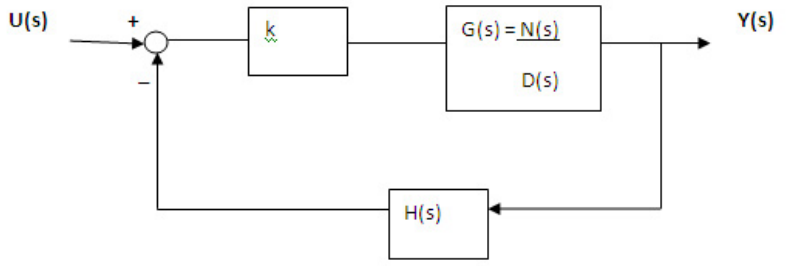

Figure 2. Block diagram representation of closed-loop linear FO system.

\section{STABILITY OF FRACTIONAL-ORDER SYSTEMS}

The stability analysis is important in control theory. Recently, there has been some advances in control theory of fractional differential systems for stability. In the FO systems the delay differential equation order is non-integer which 
makes it difficult to evaluate the stability by finding its roots or by using other algebraic methods. The stability of FO systems using polynomial criteria (e.g Routh's or Jury's type) is not possible due to the fractional powers. A generalization of the Routh-Hurwitz criterion used for stability analysis for fractional-order systems is presented in [12]. However, this method is very complicated. The geometric methods such as Nyquist type can be used for the stability check in the BIBO sense (bounded-input bounded-output). Root locus is another geometric method that can be used for analysis for FO systems [11], [14]. Also, for linear fractional differential systems of finite dimensions in state-space form, stability can be investigated. The stability of a linear fractional differential equation either by transforming the $s$-plane to the $F$-plane $\left(F=s^{\alpha}\right)$ or to the $w$-plane $\left(w=s^{1 / v}\right)$, is explained in [13]. The robust stability analysis of a Fractional-order Interval Polynomial (FOIP) family is presented in [15] and [16].

\section{A. Stability using Riemann surfaces}

The study of the stability of FO systems can be carried out by obtaining the solutions of the differential equations that characterize them. To carry out this study it is necessary to remember that a function of the type

$$
a_{n} s^{\alpha_{n}}+a_{n-1} s^{\alpha_{n-1}}+\ldots+a_{0} s^{\alpha_{0}}
$$

with $\alpha_{i} \in R^{+}, i=1, \ldots, n$ is a multi-valued function of the complex variable $s$ whose domain can be seen as a Riemann surface of a number of sheets. The principal sheet is defined by $-\pi<\arg (s)<\pi$. In the case of $\alpha_{i} \in Q^{+}$, that is, $\alpha=1 / v, v$ being a positive integer, the $v$ sheets of the Riemann surface are determined by,

$$
s=|s| e^{j \phi}, \quad(2 k+1) \pi<\phi<(2 k+3) \pi,
$$

$k=-1,0, \ldots, v-2$.

where $k=-1$ is the principal Riemann sheet. These sheets are transformed to another plane called $w$-plane with the relation $w=s^{\alpha}$. The regions of these sheets on the $w$-plane can be defined by :

$$
w=|w| e^{j \theta}, \alpha(2 k+1) \pi<\theta<\alpha(2 k+3) \pi,
$$

Thus, an equation of the type (8) which in general is not a polynomial, will have an infinite number of roots, among which only a finite number of roots will be on the principal sheet of the Riemann surface. The roots which are in the secondary sheets of the Riemann surface are iso-damping and only the roots that are in the principal sheet of the Riemann surface are responsible for a different dynamics: damped oscillation, oscillation of constant amplitude, oscillation of increasing amplitude. For the case of systems, whose characteristic equation is a polynomial of the complex variable $w=s^{\alpha}$ the stability condition is expressed as [6],

$$
\left|\arg \left(w_{i}\right)\right|>\frac{\alpha \pi}{2},
$$

where $w_{i}$ are the roots of the characteristic polynomial in $w$. For the particular case of $\alpha=1$ the well known stability condition for linear time-invariant systems of integerorder is recovered:

$$
\left|\arg \left(w_{i}\right)\right|>\frac{\pi}{2}
$$

\section{B. Frequency Response - Bode Plot}

In general, the frequency response has to be obtained by the evaluation of the irrational-order transfer function of the FO system along the imaginary axis for $s=j \omega$, $\omega \in(0, \infty)[6]$. The frequency response can be obtained by the addition of the individual contributions of the terms of order $\alpha$ resulting,

$$
G(s)=\frac{P(s)}{Q(s)}=\frac{\prod_{k=0}^{m}\left(s^{\alpha}+z_{k}\right)}{\prod_{k=0}^{n}\left(s^{\alpha}+\lambda_{k}\right)},
$$

where $z_{k}$ and $\lambda_{k}$ are the zeros and poles respectively. For each of these term the magnitude plot will have a slope which starts at zero and tends to $\pm \alpha 20 \mathrm{~dB} / \mathrm{decade}$, and the phase plot will go from 0 to $\alpha \pi / 2$.

\section{REPRESENTATION OF FRACTIONAL-ORDER SYSTEMS}

\section{A. Laplace Transform}

In system theory, the analysis of dynamical behaviors is of- ten made by means of transfer functions. Hence introduction of the Laplace transform (LT) of fractional-order derivatives is necessary for the study. Fortunately, LT for integer-order systems can be very easily applied as an effective tool even for fractional systems [10]. Inverse Laplace transformation (ILT) is also useful for time domain representation of systems for which only the frequency response is known. The most general formula assuming zero initial conditions is the following:

$$
L\left\{\frac{d^{m} f(t)}{d t^{m}}-s^{m} L\{f(t)\} .\right.
$$

This is very useful in order to calculate the inverse Laplace transform of elementary transfer functions, such as non integer order integrators $1 / s^{m}$. 


\section{B. State-space Representation}

For linear fractional differential systems of finite dimensions in state-space form, stability is investigated [6]. Consider the commensurate-order TF defined by (7), associated with this TF, canonical state-space representations can be proposed, which are similar to the classical ones developed for IO differential equation systems.

Controllable Canonical Form : Defining the first state in terms of its Laplace transform as,

$$
X_{1}(s)=\frac{1}{\sum_{k=0}^{n} a_{k}\left(s^{\alpha}\right)^{k}} U(s),
$$

and the remaining elements of the state vector in a recursive way from this one as

$$
x_{i+1}=D_{t}^{\alpha} x_{i}, \quad i=1,2, . ., n-1,
$$

the state representation, expressed in the controllable canonical form, is given by [6],

$$
D_{t}^{\alpha} x=A x+B u,
$$

$$
\begin{aligned}
& \text { where } D_{t}^{\alpha} x=\left[\begin{array}{l}
D_{t}^{\alpha} x_{1} \\
D_{t}{ }^{\alpha} x_{2} \\
. \\
D_{t}^{\alpha} x_{n-1} \\
D_{t}^{\alpha} x_{n}
\end{array}\right] \text {, } \\
& A=\left[\begin{array}{ccccc}
0 & 1 & \cdots & 0 & 0 \\
0 & 0 & \cdots & 0 & 0 \\
0 & 0 & \cdots & 0 & 0 \\
\vdots & \vdots & \ldots & \vdots & \vdots \\
0 & 0 & \cdots & 1 & 0 \\
0 & 0 & \ldots & 0 & 1 \\
-a_{0} & -a_{1} & \cdots & -a_{n-2} & -a_{n-1}
\end{array}\right] \\
& B=\left[\begin{array}{l}
0 \\
0 \\
\vdots \\
0 \\
1
\end{array}\right], \\
& y=\left[b_{0}-b_{n} a_{0} \cdots b_{n-1}-b_{n} a_{n-1}\right]\left[\begin{array}{l}
x_{1} \\
x_{2} \\
\vdots \\
x_{n}
\end{array}\right] \text {, }
\end{aligned}
$$

where $b_{i}=0$, for $m<i \leq n$

$$
C_{0}=\left\lfloor\begin{array}{lllll}
B & A B & A^{2} B & \cdots & A^{n-1} B
\end{array}\right\rfloor
$$

Controllability criterion is that the system is controllable if and only if matrix $\mathrm{C}$ defined by (18), which is called as controllable matrix is full-rank. Rearranging the above FO state equations, the observable canonical form can be obtained with the matrices $\mathrm{A}, \mathrm{B}$ and $\mathrm{C}$ matrices. The observability condition is also same as for integer-order LTI systems.

\section{ANALYTICAL RESULTS}

Some FO systems are analyzed in this section. Their stability, step response, frequency response, and the SS representation is discussed. The analysis is done using MATLAB [20]. The standard commercially available simulation softwares cannot be used for evaluating the step, ramp, frequency response of the FO systems. Recently, in MATLAB two toolboxes dedicated to FO systems are available. They are CRONE [19] and NINTEGER toolbox [18].

\section{A. Example 1}

Consider the FO integrator system with TF of the form,

$$
G(s)=\frac{1}{s^{\alpha}}
$$

For the FO integrator if $\alpha=0.5$, then consider $w=s^{0.5}$, hence $\tilde{G}(w)=\frac{1}{w}$

The system with the above function has one open-loop pole at origin. The Riemann surface of the function $w=s^{1 / v}$ has two Riemann sheets.

$$
\begin{aligned}
& \text { Now if } \alpha=1.5, \text { and consider } w=s^{0.5} \text {, then } \\
& \tilde{G}(w)=\frac{1}{w^{3}}
\end{aligned}
$$

The system with the above TF has three open-loop poles at origin.

Step Response: The system transfer function is,

$$
\frac{Y(s)}{U(s)}=\frac{1}{s^{\alpha}},
$$

Consider step input, $U(s)=1 / s$,

$$
Y(s)=\frac{1}{s s^{\alpha}}=\frac{1}{s^{\alpha+1}} .
$$

Taking inverse Laplace transform of the equation we get

$$
y(t)=\frac{t^{\alpha}}{\Gamma(\alpha+1)} .
$$


The Fig.(3) shows the step response of the system for $\alpha=0.1,0.5,0.8,1$ and 1.5 .

Frequency Response: Put $s=j \omega$ in the system function given by (19) we can plot the magnitude and phase plots. The magnitude and phase plot of the system for $\alpha=0.1$, $0.5,0.8$, and 1 is plotted as shown in the Fig.(4). From the above response we can conclude that:

1) The magnitude has a constant slope of $-20 \alpha \mathrm{dB} / \mathrm{dec}$ ade.

2) The phase plot is a horizontal line at $-\alpha \pi / 2$.

\section{B. Example 2}

Consider the incommensurate system given by the following transfer function [6] [7]

$$
G(s)=\frac{1}{0.8 s^{2.2}+0.5 s^{0.9}+1} .
$$

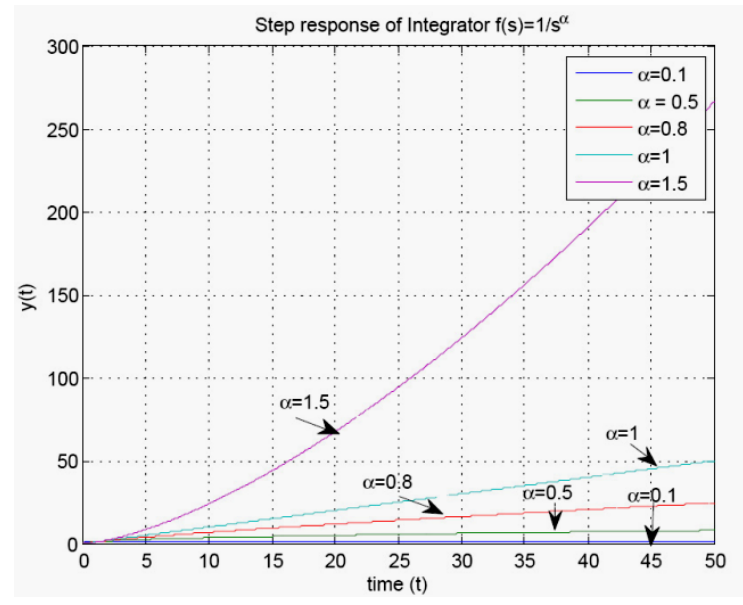

Figure 3. Step response of Example (1)

The system given in the equation can be written as

$$
G(s)=\frac{1}{0.8 s^{\left(\frac{1}{10}\right) 22}+0.5 s^{\left(\frac{1}{10}\right) 9}+1} .
$$

Consider $w=s^{\frac{1}{10}}$ the system has 10 Riemann sheets.

$$
\tilde{G}(w)=\frac{1}{0.8 w^{22}+0.5 w^{9}+1} .
$$

The open-loop poles and their appropriate arguments of the system are shown in table I. Fig.(5) gives the pole-zero plot of the open-loop system.
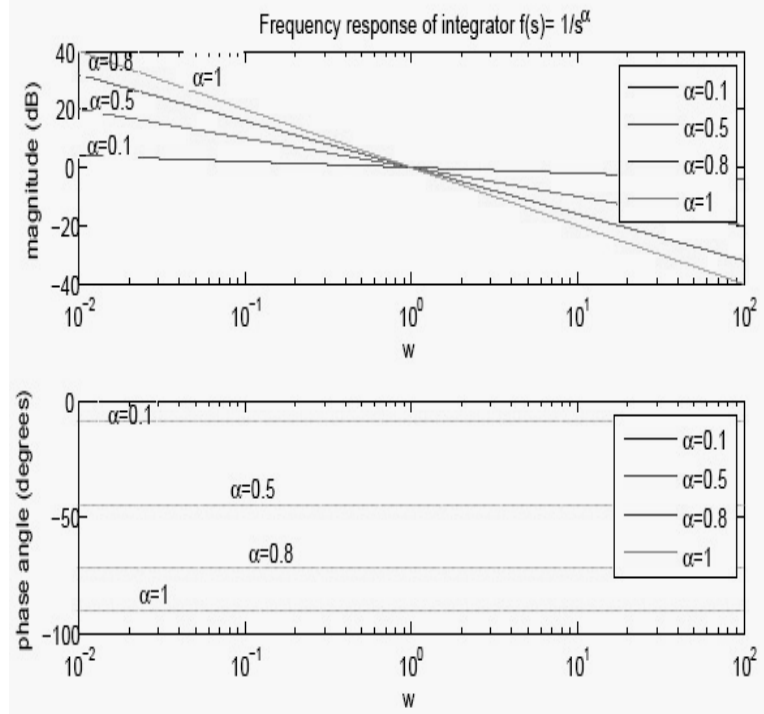

Figure 4. Frequency response of Example (1) for different values of $\alpha$

Physical significant roots are in the first Riemann sheet, which is expressed by relation $-\pi / v<\phi<\pi / v$, where $\phi=\arg (w)$.

In this example complex conjugate roots in first Riemann sheet are $w_{21,22}=1.0045 \pm 0.1684 j$, $\left|\arg \left(w_{21,22}\right)\right|=0.1661$, which satisfy conditions $\left|\arg \left(w_{21,22}\right)\right|>\pi / 2 v=\pi / 20$ is as shown in Pole-zero plot shown in Fig.(5).

TABLE I. OPEN LOOP POLES AND CORRESPONDING ARGUMENTS OF EXAMPLE (2)

\begin{tabular}{|r|r|}
\hline \multicolumn{1}{|c|}{ Poles } & Arguments in radians \\
\hline $\mathrm{w}_{1,2}=-0.9970 \pm \mathrm{j} 0.1182$ & $\left|\arg \left(\mathrm{w}_{1,2}\right)\right|=3.023$ \\
\hline $\mathrm{w}_{3,4}=-0.9297 \pm \mathrm{j} 0.4414$ & $\left|\arg \left(\mathrm{w}_{3,4}\right)\right|=2.698$ \\
\hline $\mathrm{w}_{5,6}=-0.7465 \pm \mathrm{j} 0.6420$ & $\left|\arg \left(\mathrm{w}_{5,6}\right)\right|=2.431$ \\
\hline $\mathrm{w}_{7,8}=-0.5661 \pm \mathrm{j} 0.8633$ & $\left|\arg \left(\mathrm{w}_{7,8}\right)\right|=2.151$ \\
\hline $\mathrm{w}_{9,10}=-0.259 \pm \mathrm{j} 0.9625$ & $\left|\arg \left(\mathrm{w}_{9,10}\right)\right|=1.834$ \\
\hline $\mathrm{w}_{11,12}=-0.0254 \pm \mathrm{j} 1.0111$ & $\left|\arg \left(\mathrm{w}_{11,12}\right)\right|=1.595$ \\
\hline $\mathrm{w}_{13,14}=0.3080 \pm \mathrm{j} 0.9772$ & $\left|\arg \left(\mathrm{w}_{11,12}\right)\right|=1.265$ \\
\hline $\mathrm{w}_{15,16}=0.5243 \pm \mathrm{j} 0.8359$ & $\left|\arg \left(\mathrm{w}_{15,16}\right)\right|=1.010$ \\
\hline $\mathrm{w}_{17,18}=0.7793 \pm \mathrm{j} 0.6795$ & $\left|\arg \left(\mathrm{w}_{17,18}\right)\right|=0.717$ \\
\hline $\mathrm{w}_{19,20}=0.9084 \pm \mathrm{j} 0.3960$ & $\left|\arg \left(\mathrm{w}_{19,20}\right)\right|=0.411$ \\
\hline $\mathrm{w}_{21,22}=1.0045 \pm \mathrm{j} 0.1684$ & $\left|\arg \left(\mathrm{w}_{21,22}\right)\right|=0.1661$ \\
\hline
\end{tabular}




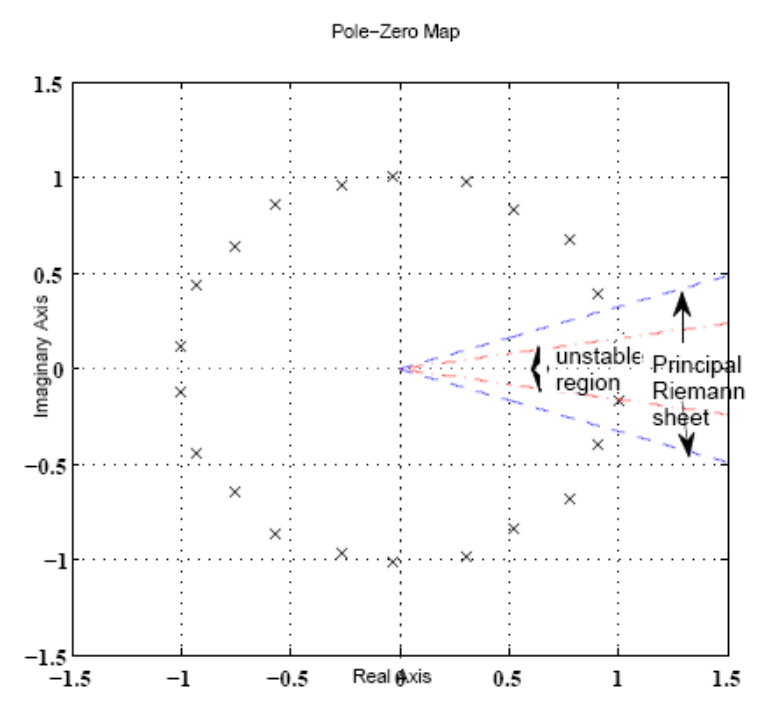

Figure 5. Open loop pole-zero plot of Example (2)

The roots in first Riemann sheet satisfy the stability criteria, hence the system is stable. Other roots of the system lie in secondary Riemann sheets. The first Riemann sheet is transformed from $s$ plane to $w$-plane as follows:

$$
\begin{gathered}
-\pi / 10<\arg (w)<\pi / 10, \text { and } \\
-\pi<10 \arg (w)<\pi .
\end{gathered}
$$

Therefore from this consideration angle obtained is

$$
|\arg (s)|=10|\arg (w)| .
$$

The closed loop poles are given in table II and are plotted in Fig.(6).

TABLE II. CLOSED LOOP POLES AND CORRESPONDING ARGUMENTS OF EXAMPLE (2)

\begin{tabular}{|r|r|}
\hline \multicolumn{1}{|c|}{ Poles } & Arguments in radians \\
\hline $\mathrm{w}_{1,2}=-1.0298 \pm \mathrm{j} 0.1311$ & $\left|\arg \left(\mathrm{w}_{1,2}\right)\right|=3.015$ \\
\hline $\mathrm{w}_{3,4}=-0.9557 \pm \mathrm{j} 0.4483$ & $\left|\arg \left(\mathrm{w}_{3,4}\right)\right|=2.703$ \\
\hline $\mathrm{w}_{5,6}=-0.7764 \pm \mathrm{j} 0.6694$ & $\left|\arg \left(\mathrm{w}_{5,6}\right)\right|=2.430$ \\
\hline $\mathrm{w}_{7,8}=-0.5776 \pm \mathrm{j} 0.8863$ & $\left|\arg \left(\mathrm{w}_{7,8}\right)\right|=2.148$ \\
\hline $\mathrm{w}_{9,10}=-0.2768 \pm \mathrm{j} 0.9956$ & $\left|\arg \left(\mathrm{w}_{9,10}\right)\right|=1.842$ \\
\hline $\mathrm{w}_{11,12}=-0.0173 \pm \mathrm{j} 1.0430$ & $\left|\arg \left(\mathrm{w}_{11,12}\right)\right|=1.587$ \\
\hline $\mathrm{w}_{13,14}=0.3099 \pm \mathrm{j} 1.0055$ & $\left|\arg \left(\mathrm{w}_{11,12}\right)\right|=1.271$ \\
\hline $\mathrm{w}_{15,16}=0.5488 \pm \mathrm{j} 0.8676$ & $\left|\arg \left(\mathrm{w}_{15,16}\right)\right|=1.006$ \\
\hline $\mathrm{w}_{17,18}=1.0348 \pm \mathrm{j} 0.1653$ & $\left|\arg \left(\mathrm{w}_{17,18}\right)\right|=0.1584$ \\
\hline $\mathrm{w}_{19,20}=0.9412 \pm \mathrm{j} 0.4170$ & $\left|\arg \left(\mathrm{w}_{19,20}\right)\right|=0.417$ \\
\hline $\mathrm{w}_{21,22}=0.7989 \pm \mathrm{j} 0.6953$ & $\left|\arg \left(\mathrm{w}_{21,22}\right)\right|=0.7162$ \\
\hline
\end{tabular}

pole-zero plot in w-plane

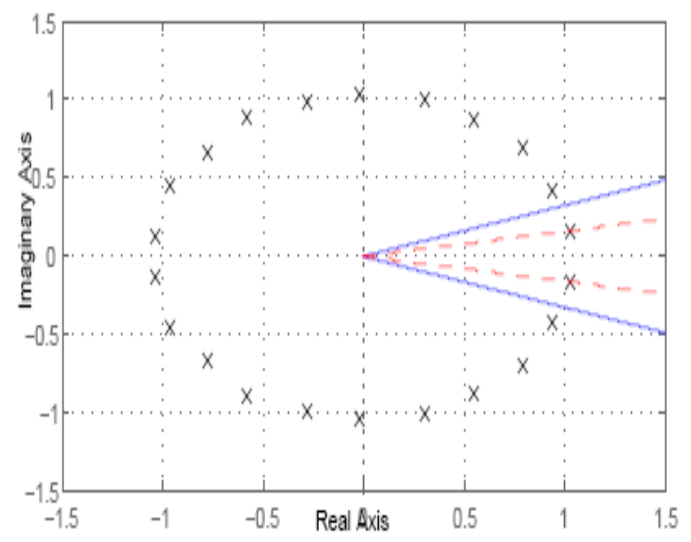

Figure 6. Closed loop pole-zero plot of Example (2)

Step Response: The system TF is,

$$
\frac{Y(s)}{U(s)}=\frac{1}{0.8 s^{2.2}+0.5 s^{0.9}+1} .
$$

For step response of the system, $U(s)=1 / s$.

Calculating the residues and poles by partial fractions are shown in table III.

\section{TABLE III. RESIDUES AND CORRESPONDING POLES}

\begin{tabular}{|r|r|}
\hline \multicolumn{1}{|c|}{ Residues } & \multicolumn{1}{c|}{ Poles } \\
\hline$-0.0264 \pm \mathrm{j} 0.0209$ & $0.7793 \pm \mathrm{j} 0.6796$ \\
\hline $0.0147 \pm \mathrm{j} 0.0313$ & $-0.5662 \pm \mathrm{j} 0.8633$ \\
\hline $0.0355 \pm \mathrm{j} 0.0079$ & $-0.9298 \pm \mathrm{j} 0.4415$ \\
\hline$-0.0006 \pm \mathrm{j} 0.0391$ & $0.3080 \pm \mathrm{j} 0.9772$ \\
\hline$-0.0422 \pm \mathrm{j} 0.0068$ & $1.0045 \pm \mathrm{j} 0.1684$ \\
\hline$-0.0142 \pm \mathrm{j} 0.0447$ & $-0.0254 \pm \mathrm{j} 1.0112$ \\
\hline $0.0467 \pm \mathrm{j} 0.0210$ & $-0.9970 \pm \mathrm{j} 0.1182$ \\
\hline $0.0271 \pm \mathrm{j} 0.0477$ & $-0.2597 \pm \mathrm{j} 0.9625$ \\
\hline$-0.0476 \pm \mathrm{j} 0.0323$ & $0.9085 \pm \mathrm{j} 0.3960$ \\
\hline$-0.0369 \pm \mathrm{j} 0.0464$ & $0.5243 \pm \mathrm{j} 0.8360$ \\
\hline $0.0441 \pm \mathrm{j} 0.0409$ & $-0.7466 \pm \mathrm{j} 0.6420$ \\
\hline
\end{tabular}

Using inverse Laplace transform [6],

$$
L^{-1}\left\{\sum_{i=1}^{n} \frac{r_{i}}{s\left(s^{\alpha}+p_{i}\right)}\right\}=\sum_{i=1}^{n} r_{i} t^{\alpha} E_{\alpha, \alpha+1}\left(-p_{i} t^{\alpha}\right)
$$


where $E_{\alpha, \beta}($.$) is the Mittag Leffler (ML) function as$ defined in Section II, $r_{i}$ are the residues and $p_{i}$ are the corresponding poles for $i=1$ to 22 .

To plot step response we have used the MATLAB subroutine 'mlf()' developed by Podlubny [21]. The step response plot is plotted as shown in Fig.(7). The step response shows it is a underdamped system. This is obvious as the two stable poles in the principal Riemann sheet are very close to the imaginary axis in the $s$-plane. See Fig.(6) for the corresponding $w$-plane situation.

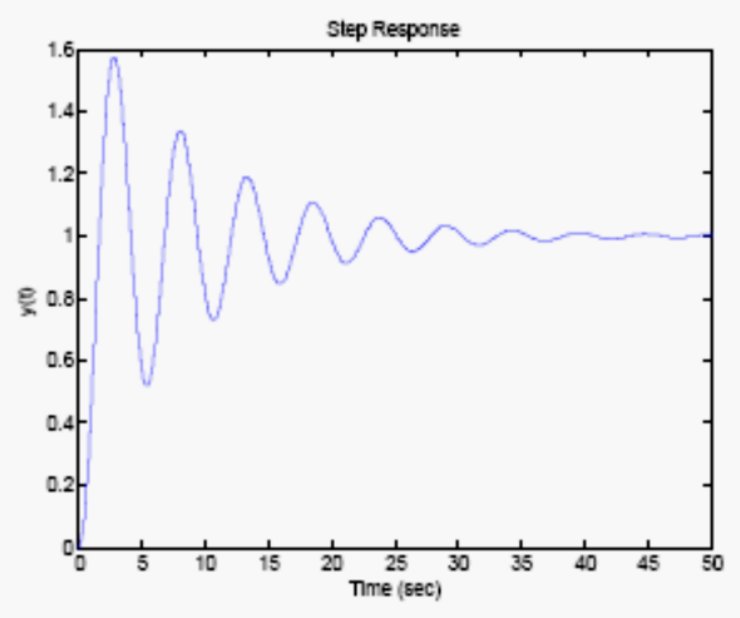

Figure 7. Step response of Example (2)

Frequency Response: Put $s=j \omega$ in the given system function. The magnitude plot and phase plot of the system using MATLAB is plotted as shown in the Fig.(8). The gain margin is $\infty$ and the phase margin is about $177^{\circ}$.
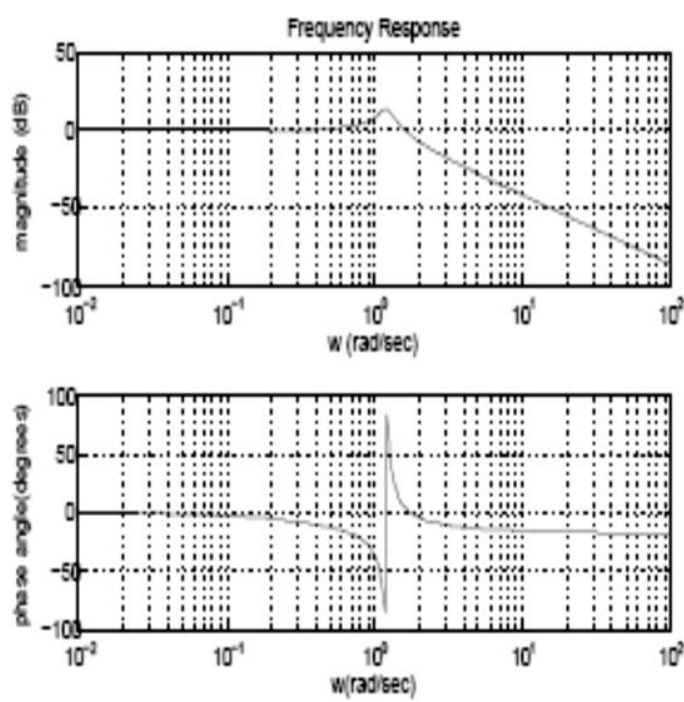

Figure 8. Frequency response of Example (2)

State-space Representation: The canonical form of the system is obtained as,

$$
\frac{Y(s)}{X(s)}=\frac{1}{0.8 s^{(0.1) 22}+0.5 s^{(0.1) 9}+1} .
$$

$$
\left(\left(s^{0.1}\right)^{22}+0.625\left(s^{0.1}\right)^{9}+1.25\right) Y(s)=1.25 X(s) .
$$

Consider input $\mathbf{u}(\mathbf{t})$ and taking inverse Laplace transform we get,

$$
D_{t}^{2.2} y(t)+0.625 D_{t}^{0.9} y(t)+1.25 y(t)=1.25 u(t),
$$

Case 1: Let $y(t)=x_{1}(t) \quad$ and

$$
D_{t}^{0.1} x_{1}(t)=x_{2}(t)
$$

In general we have $x_{i+1}=D_{t}^{0.1} x_{i}, \quad i=1,2, . ., 21$.

$$
D_{t}^{0.1} x_{22}(t)=-1.25 x_{1}(t)-0.625 x_{10}(t)+1.25 u(t),
$$

The controllable canonical form is therefore given by,

$$
\left[\begin{array}{l}
D_{t}^{0.1} x_{1}(t) \\
D_{t}^{0.1} x_{2}(t) \\
\vdots \\
D_{t}^{0.1} x_{22}(t)
\end{array}\right]
$$$$
=\left[\begin{array}{cccccc}
0 & 1 & \ldots & 0 & \ldots & 0 \\
0 & 0 & \ldots & 0 & \ldots & 0 \\
\vdots & \vdots & \vdots & \vdots & \ldots & \vdots \\
-1.25 & 0 & \cdots & -0.625 & \ldots & 0
\end{array}\right] \quad\left[\begin{array}{c}
x_{1}(t) \\
x_{2}(t) \\
\vdots \\
x_{22}(t)
\end{array}\right]
$$$$
+\left[\begin{array}{c}
0 \\
0 \\
\vdots \\
1.25
\end{array}\right] u(t)
$$

$$
y(t)=\left[\begin{array}{llll}
1 & 0 & \cdots & 0
\end{array}\right] u(t) .
$$

Case 2: Let $y(t)=x_{1}(t)$ and $D_{t}^{0.9} x_{1}(t)=x_{2}(t)$

The controllable canonical form is therefore given by,

$$
\begin{gathered}
{\left[\begin{array}{c}
D_{t}^{0.9} x_{1}(t) \\
D_{t}^{1.3} x_{2}(t)
\end{array}\right]=\left[\begin{array}{cc}
0 & 1 \\
-0.125 & -0.625
\end{array}\right]\left[\begin{array}{l}
x_{1}(t) \\
x_{2}(t)
\end{array}\right]} \\
+\left[\begin{array}{c}
0 \\
0.125
\end{array}\right] u(t) \\
\end{gathered}
$$

The controllable matrix of this system is full rank and hence the system is controllable. It is also shown that there can be no unique state space representation for a fractional-order system. In the analysis of this 
incommensurate FO system we conclude that the system is stable, controllable and observable.

\section{Example 3}

Consider the commensurate system given by the following transfer function [6] :

$$
G(s)=\frac{1}{s-2 s^{0.5}+1.25} .
$$

The system given in the equation can be written as

$$
G(s)=\frac{1}{s^{\left(\frac{1}{2}\right)^{2}-2 s^{\left(\frac{1}{2}\right) 1}+1} .}
$$

Consider $w=s^{\frac{1}{2}}$, the system has two Riemann sheets. Transforming the system onto $w$-plane we get,

$$
\tilde{G}(w)=\frac{1}{w^{2}-2 w+1.25} \text {. }
$$

The open-loop poles and their appropriate arguments of the system are shown in table IV.

\section{TABLE IV. OPEN LOOP POLES AND CORRESPONDING ARGUMENTS OF EXAMPLE 3}

\begin{tabular}{|c|c|}
\hline Poles & Arguments in radians \\
\hline $\mathrm{w}_{1,2}=1.0000 \pm \mathrm{j} 0.5000$ & $\arg \left(\mathrm{w}_{1,2}\right) \mid=0.4636$ \\
\hline
\end{tabular}

The open-loop pole-zero plot is shown in the Fig.(9). The poles lie in the unstable region $-\pi / 4<\arg (w)<\pi / 4$, and the first Riemann sheet is $-\pi / 2<\arg (w)<\pi / 2$.

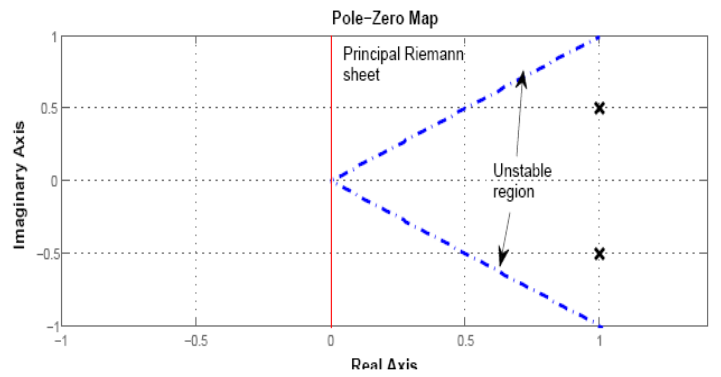

Figure 9. Open-loop pole-zero plot of Example (3)

The closed-loop poles and their appropriate arguments of the system are shown in table $\mathrm{V}$.

\section{TABLE V. CLOSED LOOP POLES AND CORRESPONDING ARGUMENTS OF EXAMPLE 3}

\begin{tabular}{|c|c|}
\hline Poles & Arguments in radians \\
\hline $\mathrm{w}_{1,2}=1.0000 \pm \mathrm{j} 1.1180$ & $\arg \left(\mathrm{w}_{1,2}\right) \mid=0.8411$ \\
\hline
\end{tabular}

The closed-loop pole-zero plot is shown in the Fig.(10). The poles are in the stable region, which implies that the closed-loop system is stable.

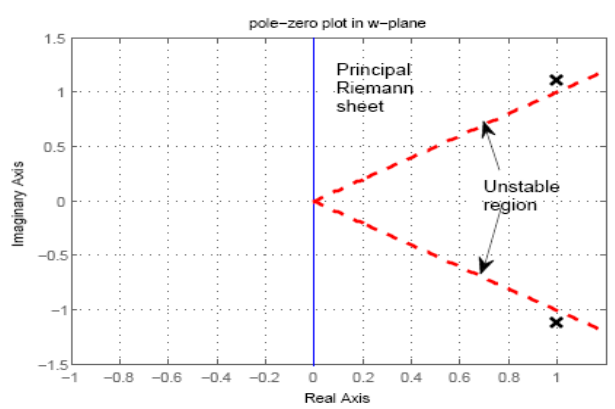

Figure 10. Closed-loop pole-zero plot of Example (3)

Step Response: The step response is obtained using invlap subroutine [23] for the closed-loop system with unity gain as shown in Fig.(11). It is observed that the ML function calculation is time consuming and may not give proper results in all the cases. In such cases they can also be plotted using invlap.m subroutine (numerical ILT) [22], [23].

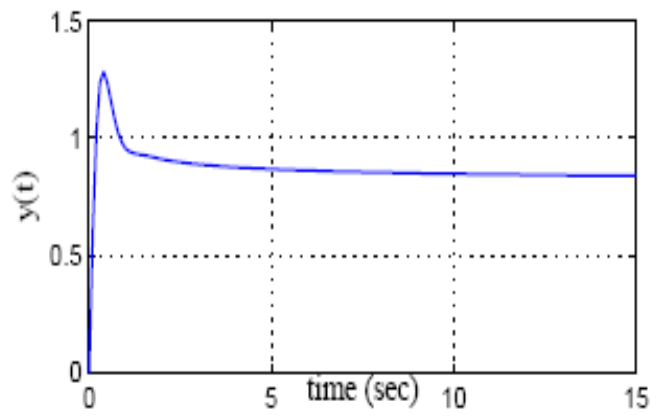

Figure 11. Step response of Example (3)

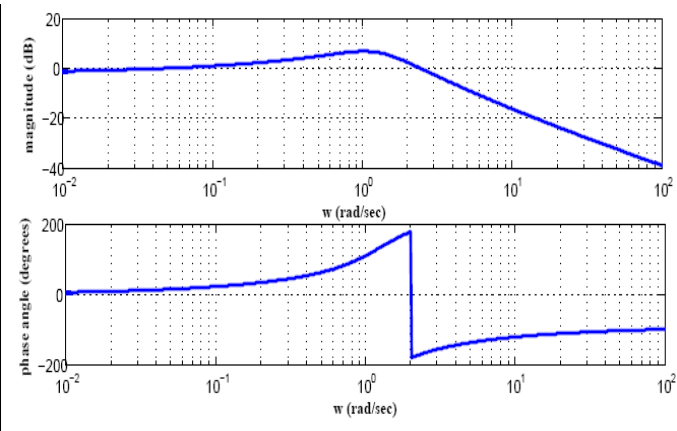

Figure 12. Frequency response of Example (3)

Frequency Response: Put $s=j \omega$ in the given system function. The magnitude plot and phase plot of the system using MATLAB is plotted as shown in the Fig.(12). The gain margin is $\infty$ and the phase margin is about $193^{\circ}$. This shows that the system is stable with a wide range of gain and phase margins.

State-space Representation: The canonical form of the system is obtained as, 


$$
\frac{Y(s)}{X(s)}=\frac{1}{s-2 s^{0.5}+1.25},
$$

Using the procedure as given in Section VI, we get,

$$
\begin{aligned}
& D_{t}^{0.5}\left[\begin{array}{l}
x_{1}(t) \\
x_{2}(t)
\end{array}\right]=\left[\begin{array}{cc}
0 & 1 \\
-1.25 & 2
\end{array}\right]\left[\begin{array}{l}
x_{1}(t) \\
x_{2}(t)
\end{array}\right]+\left[\begin{array}{l}
0 \\
1
\end{array}\right] u(t) \\
& y(t)=\left[\begin{array}{ll}
1 & 0
\end{array} u(t) .\right.
\end{aligned}
$$

Where $A=\left[\begin{array}{cc}0 & 1 \\ -1.25 & 2\end{array}\right], B=\left[\begin{array}{l}0 \\ 1\end{array}\right], C=[1$

The system is found to be controllable and observable. From the open-loop and closed-loop pole-zero plots, and the gain margin and phase margin it can be concluded that the system is stable in the closed-loop configuration.

\section{Example 4}

Consider the commensurate system given by the following open loop transfer function [11].

$$
G(s)=\frac{s^{0.5}-1}{s^{2}-3 s^{1.5}-2 s+2 s^{0.5}+12} .
$$

The system given in the equation can be written as

$$
G(s)=\frac{s^{0.5}-1}{\left.s^{\left(\frac{1}{2}\right) 4}-3 s^{\left(\frac{1}{2}\right)}\right)^{3}-2 s^{\left(\frac{1}{2}\right) 2}+2 s^{\left(\frac{1}{2}\right)}+12} .
$$

Consider $w=s^{\frac{1}{2}}$, the system has two Riemann sheets. Transforming the system onto $w$-plane we get,

$$
\tilde{G}(w)=\frac{w-1}{w^{4}-3 w^{3}-2 w^{2}+2 w+12} .
$$

The open-loop poles, zeros and their appropriate arguments of the system are shown in table VI.

TABLE VI. OPEN LOOP POLES AND CORRESPONDING ARGUMENTS OF EXAMPLE 4

\begin{tabular}{|c|c|}
\hline Poles & Arguments in radians \\
\hline $\mathrm{w}_{1}=3.0000$ & $\left|\arg \left(\mathrm{w}_{1}\right)\right|=0.0000$ \\
\hline $\mathrm{w}_{2}=2.0000$ & $\left|\arg \left(\mathrm{w}_{2}\right)\right|=0.0000$ \\
\hline $\mathrm{w}_{3,4}=-1.0000 \pm \mathrm{j} 1.0000$ & $\left|\arg \left(\mathrm{w}_{3,4}\right)\right|=2.3562$ \\
\hline Zeros & $\operatorname{Arguments}$ in radians \\
\hline $\mathrm{w}_{5}=1.0000$ & $\left|\arg \left(\mathrm{w}_{5}\right)\right|=0.0000$ \\
\hline
\end{tabular}

The open-loop pole-zero plot of the system in the $w$ plane is as shown in the Fig.(13). It shows the unstable region $-\pi / 4<\arg (w)<\pi / 4$, and the first Riemann sheet is $-\pi / 2<\arg (w)<\pi / 2$. Also there are 2 poles and 1 zero in the unstable region and 2 poles in the stable region on the second Riemann sheet.

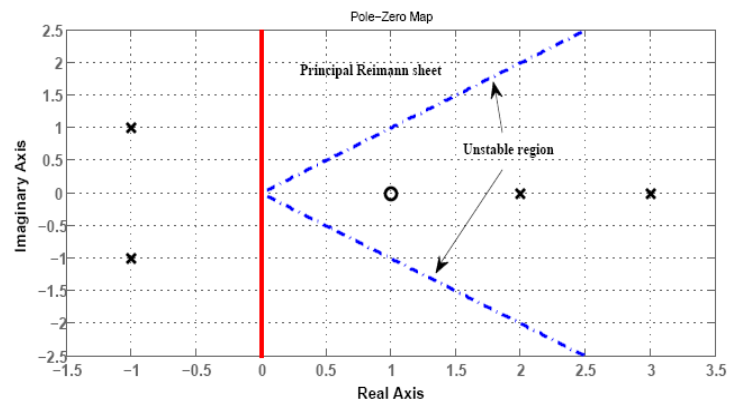

Figure 13. Open-loop pole-zero plot of Example (4)

The closed-loop poles, zeros and their appropriate arguments of the system are shown in table VII.

TABLE VII. CLOSED LOOP POLES AND CORRESPONDING ARGUMENTS OF EXAMPLE 4

\begin{tabular}{|c|c|}
\hline Poles & Arguments in radians \\
\hline $\mathrm{w}_{1}=2.867$ & $\left|\arg \left(\mathrm{w}_{1}\right)\right|=0.0000$ \\
\hline $\mathrm{w}_{2}=2.1183$ & $\left|\arg \left(\mathrm{w}_{2}\right)\right|=0.0000$ \\
\hline $\mathrm{w}_{3,4}=-0.9915 \pm \mathrm{j} 0.9109$ & $\left|\arg \left(\mathrm{w}_{3}, 4\right)\right|=2.3985$ \\
\hline Zeros & $\operatorname{Arguments}$ in radians \\
\hline $\mathrm{w}_{5}=1.0000$ & $\left|\arg \left(\mathrm{w}_{5}\right)\right|=0.0000$ \\
\hline
\end{tabular}

The pole-zero plot of the closed-loop system in the $w$ plane is as shown in the Fig.(14). There are 2 poles and 1 zero in the unstable region and 2 poles in the stable region on the second Riemann sheet which is similar to the case of openloop system.

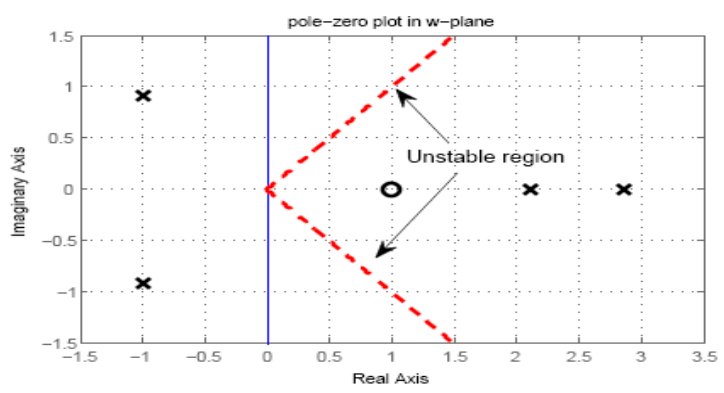

Figure 14. Closed-loop pole-zero plot of Example (4)

Step Response: The closed-loop step response is obtained using invlap subroutine [23] for unity gain as shown in Fig.(15). It shows that the system is unstable.

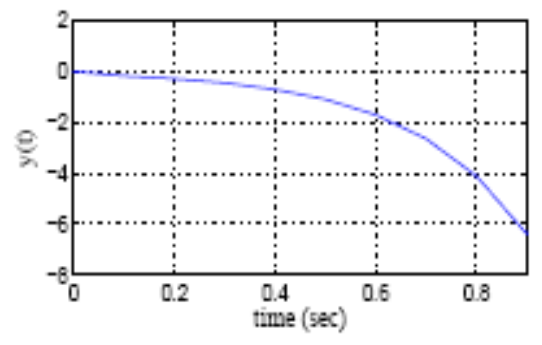

Figure 15. Step response of Example (4) 
Frequency Response: Put $s=j \omega$ in the given system function. The magnitude plot and phase plot of the system using MATLAB is plotted as shown in the Fig.(16). The gain margin is about $35 \mathrm{~dB}$ and phase margin is $\infty$.
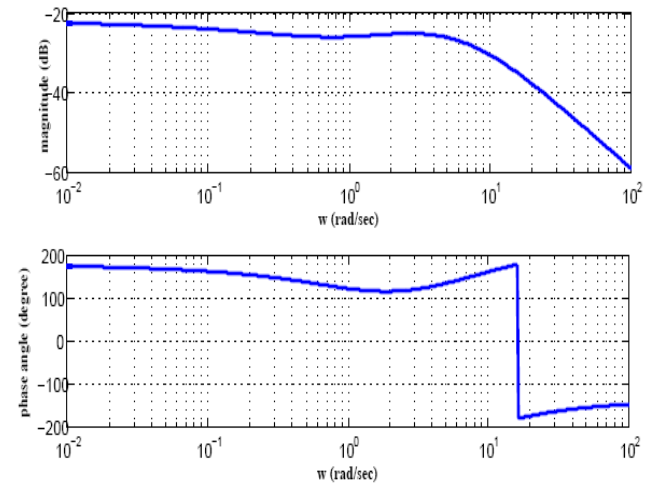

Figure 16. Frequency response of Example (4)

State-space Representation: The canonical form of the system is obtained as,

$$
\frac{Y(s)}{X(s)}=\frac{s^{0.5}-1}{s^{2}-3 s^{1.5}-2 s+2 s^{0.5}+12},
$$

Using the procedure as given in Section VI, we get,

$$
D_{t}^{0.5}\left[\begin{array}{l}
x_{1}(t) \\
x_{2}(t) \\
x_{3}(t) \\
x_{4}(t)
\end{array}\right]=\left[\begin{array}{cccc}
0 & 1 & 0 & 0 \\
0 & 0 & 1 & 0 \\
0 & 0 & 0 & 1 \\
-12 & -2 & 2 & 3
\end{array}\right]\left[\begin{array}{c}
x_{1}(t) \\
x_{2}(t) \\
x_{3}(t) \\
x_{4}(t)
\end{array}\right]
$$$$
+\left[\begin{array}{l}
0 \\
0 \\
0 \\
1
\end{array}\right] u(t)
$$

$$
y(t)=\left[\begin{array}{llll}
-1 & 1 & 0 & 0
\end{array}\right]\left[\begin{array}{l}
x_{1}(t) \\
x_{2}(t) \\
x_{3}(t) \\
x_{4}(t)
\end{array}\right]+[0] u(t)
$$

Where $A=\left[\begin{array}{cccc}0 & 1 & 0 & 0 \\ 0 & 0 & 1 & 0 \\ 0 & 0 & 0 & 1 \\ -12 & -2 & 2 & 3\end{array}\right], B=\left[\begin{array}{l}0 \\ 0 \\ 0 \\ 1\end{array}\right]$,
$C=[-1 \quad 1 \quad$
-1

The system is found to be controllable and observable. From the open-loop and closed loop pole-zero plots, step response we conclude that the system is unstable.

\section{CONCLUSION}

The fractional-order models of real systems are more adequate than the usually used integer order models. At the same time fractional-order controllers provide better performance in comparison to integer order controllers. The most important features such as stability, controllability, observability, stability margins of linear fractional-order systems are studied during the work. They are discussed using Bode diagrams, time response, state space representation. The time and frequency domain analysis of fractional-order systems is found to be similar to that of integer order systems.

\section{ACKNOWLEDGMENT}

Our thanks to all those who have directly or indirectly helped us in completion of this work. Special thanks to our family members for their support and encouragement.

\section{REFERENCES}

[1] T.F. Nonnenmacher, W.G. Glockle, A Fractional Model for mechanical stress relaxation, $3^{\text {rd }}$ ed. Phil Maglett: 64(2):89-93, 1991

[2] S. Westerlund and L. Ekstam, Capacitor theory, 3rd .IEEE Trans Dielectrics and Insulation; 1(5), 826-39, 1994.

[3] R.L. Bagley, R.A. Calico, Fractional order state equations for the control of viscoelastic structures, 3rd ed. J Guid Control Dyn; 14(2):304$11,1991$.

[4] I. Podlubny, Fractional Differential Equations, Mathematics in Science and Engineering, 3rd ed. San Diego: Academic Press, 1999.

[5] W. LePage, Complex Variables and the Laplace Transform for Engineers, 3rd ed. McGraw-Hill, New York, NY, USA, series: International Series in Pune and Applied Mathematics 1961.

[6] C. Monje , Y. Chen, B. Vinagre, D. Xue and V. Feliu, Fractional-order Systems and Controls Fundamentals and Applications, Springer London Dordrecht Heidelberg New York, 2010.

[7] I. Podlubny, Fractional-order systems and P I $\alpha \mathrm{D}^{\beta}$-controllers, IEEE Transactions on Automatic Control, vol. 44, no. 1, pp. 208-214, 1999.

[8] R. Hilfer, Ed., Applications of Fractional Calculus in Physics, World Scientific, River Edge, NJ, USA, 2000.

9] K. B. Oldham and J. Spanier, The Fractional Calculus: Theory and Applications of Differentiation and Integration to Arbitrary Order, Academic Press, New York, NY, USA, 1974.

[10] R. Caponetto, G. Dongola, L.Fortuna and I.Petras, Fractional Order Systems Modeling and Control Applications, World Scientific Publishing Co. Pte. Ltd. vol.72, series A.

[11] F. Merrikh-Bayat and M. Afshar, Extending the Root-Locus Method to Fractional-Order Systems, Journal of Applied Mathematics, Hindawi Publishing Corporation vol.2008.

[12] M. Ikeda and S. Takahashi, Generalization of Routh's algorithm and stability criterion for non-integer integral system, Electronics and Communications in Japan vol.22, no.2, pp. 41-50, 1977.

[13] A.G. Radwan, A.M. Soliman, A.S. Elwakil and A. Sedeek, On the stability of linear systems with fractional-order elements, Chaos, Solitons and Fractals 40, 23172328, 2009.

[14] T. Machado, J. A., Root Locus of Fractional Linear Systems, Communications in Nonlinear Science and Numerical Simulation (2011) doi 10.1016/j.cnsns.2011.01.020 
[15] N. Tan, O. F. Ozguven, M. M. Ozyetkin, Robust stability analysis of fractional order interval polynomials, ISA Transactions 48 (2009) $166-172$.

[16] H. Kang, H. S. Lee, J. W. Bae, Robust Stability Analysis of Commensurate Fractional Order Interval Polynomials, ISECS International Colloquium on Computing, Communication, Control, and Management,2009.

[17] J. Machado, V. Kiryakova, F. Mainardi, Recent history of fractional calculus, In: Commun Nonlinear Sci Numer Simulat, Elsvier, 2010.

[18] D. Valrio and S da Costa, Ninteger: A non-integer control toolbox for MatLab, In: Fractional derivatives and applications, Bordeaux, 2004.

[19] P. Melchior, B. Orsoni, O. Lavialle, A. Oustaloup, The CRONE toolbox for Matlab: fractional path planning design in robotics, Laboratoire dAutomatique et de Productique (LAP), 2001.
[20] The MathWorks Inc. MATLAB Control System Toolbox, Users Guide, 2000.

[21] I. Podlubny, Mittag-Leffler function, onlinehttp://www.mathworks.com/matlabcentral/fileexchange/8738, 2005.

[22] H. Shenga, Y. Lib, and Y. Chen, Application of Numerical Inverse Laplace Transform Algorithms in Fractiona Calculus, Proceedings of FDA10. The 4th IFAC Workshop Fractional Differentiation and its Applications. Badajoz, Spain, October 18-20, 2010.

[23] K. Hollenbeck, "Invlap.m": A Matlab function for numerical inversion of Laplace transforms by the de hoog algorithm, http://www.isva.dtu.dk/staff/karl/invlap.htm. 1998.

[24] R.L. Magin, Fractional Calculus in Bioengineering,Begell House, 2006.

[25] R. Gorenflo, F. Mainardi, A. Carpintieri, Fractional calculus: Integral and differential equations of fractional order, Fractals and Fractional Calculus in Continuum Mechanics. Springer Verlag, 1997. 